\title{
Polen - das zukünftige Zentrallager in Europa?
}

\author{
Ireneusz Fechner, Tomasz Janiak
}

\section{Zusammenfassung}

Die Rekonfigurierung des Distributionsnetzes großer internationalen Produktions- und Handelsunternehmen hat die Verschiebung von Distributionszentren in Richtung Osten, darunter Polen, zur Folge. Darüber hinaus spielt Polen eine wichtige Rolle in den paneuropäischen Transportkorridoren. Das Ziel dieses Betrages ist es, den gegenwärtigen Stand bei der Entwicklung von Logistikimmobilien in Polen aufzuzeigen.

\section{Abstract}

Poland - the future Central Warehouse in Europe ? The Rearranging of Distribution Networks of big international production and trade companies causes the relocating of distribution centers towards East Europe, including Poland. Furthermore, Poland plays an important role in the Pan-European transport corridors. This paper presents the current development of logistics facilities in Poland.

\section{Einleitung}

Polen hat eine strategische Bedeutung für die Entwicklung von Zentraleuropa. Es ist das Land mit dem größten Markt unter den neuen EU-Mitgliedern. Durch die damit verbundene Öffnung des europäischen Binnenmarktes wurden neue Wettbewerbsbedingungen geschaffen. Dies bedingt aber auch einen wachsenden Anteil Polens an den internationalen Lieferketten. Mit dem Beitritt Polens und anderer MOE Länder begann ein Prozess der Rekonfigurierung der Distributionsnetze großer internationalen Produktions- und Handelsunternehmen. Dieser Umstand hat die Verschiebung von Distributionszentren in Richtung Osten zur Folge.

\section{Vorteil der geographischen Lage Polens}

Aus der Perspektive der globalen Wirtschaft ist die geographische Lage Polens zwischen der Europäischen Union und Russland, Ukraine, China und anderen asiatischen Ländern eine Chance für einen sehr attraktiven Logistikstandort. Nur über Polen verlaufen pan-europäische Transportkorridore, die den Transit zwischen diesen Wirtschaftsräumen ermöglichen. Darüber hinaus verlaufen durch dieses Land Transportkorridore, die Südeuropa und Mittelosteuropa mit den Baltikumstaaten Litauen, Lettland, Estland und Finnland verbinden. Die östliche Grenze Polens ist die östliche Außengrenze der Europäischen Union und zugleich die Grenze des europäischen Zollgebietes der Gemeinschaft. Der pan-europäische Transportkorridor VI und die Seehäfen in Gdynia und Gdansk sowie die Häfen in Szczecin und Swinoujscie verbinden Polen mit dem Wirtschaftsräumen der Ostseeregion, deren Integration im Rahmen des europäischen Interreg-Programms stattfindet.

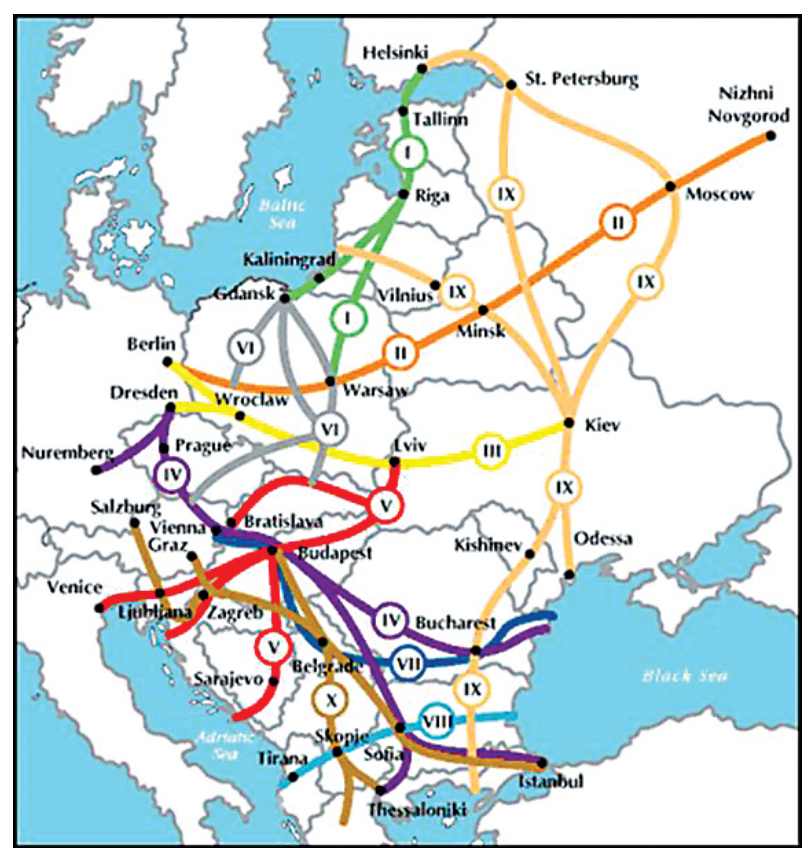

Abb. 1: Europäische Transportkorridore, die über Polen verlaufen. Quelle: bulletin.rec.org/bull103/corridors.html 
Transit als solcher weckt zwar keinen Bedarf an Logistikimmobilien, aber auf Grund der Tatsache, dass Polens östliche Grenze die Außengrenze des Zollgebietes bildet, kann angenommen werden, dass ein Teil des Warenflusses, der in den EU-Raum von Osten eingeführt wird, in den polnischen Lagerhäusern temporär gelagert wird.

\section{Vorteil der Wirtschaftlage Polens}

Die Wirtschaft Polens entwickelt sich seit einigen Jahren dynamischer als die Wirtschaften der »alten « EU15. Aufgrund der konkurrenzfähigen Arbeitskosten im Vergleich zu Westeuropa steigt die internationale Kooperation im Produktionsbereich. Aus diesem Umstand resultieren die zunehmende Anzahl ausländischer Industrieunternehmen in Polen, insbesondere aus der Automobilbranche und der Unterhaltungselektronik, sowie das Wachstum des polnischen Marktes für Logistikdienstleistungen, der 2007 den Wert von 120 Mrd. PLN überschritten hat.

\section{Polen als Markt für Lagerflächen}

Die aufgeführten günstigen Rahmenbedingungen hatte einen Anstieg der Nachfrage an modernen Lagerflä- chen, die vor dem Jahr 1990 praktisch nicht zugänglich waren, zur Folge. Die 1989 begonnene Änderung des Wirtschaftssystems hatte auch eine Veränderung des nationalen Logistiksystems zur Folge; ein Markt für Logistikimmobilien (Abb. 3) begann sich zu entwickeln. Komplexe Güterverkehrszentren (GVZ), die sich von Lagerzentren durch das Vorhandensein intermodaler Transportlösungen unterscheiden, entwickeln sich jedoch nur langsam. Der Markt für moderne Lagerflächen ist momentan von monomodalen Lagerzentren geprägt. Der Bedarf an modernen Lagerflächen wird in Polen gegenwärtig hautsächlich durch die Errichtung von Lagerobjekten durch Bauherren (Developer) ohne zusätzliche intermodale Infrastruktur erfüllt, da Polen nie ein nationales Programm für die Gründung von GVZ hatte. Alle Initiativen in diesem Bereich fanden keine Unterstützung seitens des Staates und der öffentlichen Finanzen (Abb. 2).

Daher ist die Gründung von folgenden vier Logistikzentren (GVZ):

1. Śląskie Centrum Logistyki S.A. in Gliwice,

2. Wielkopolskie Centrum Logistyczne Konin-Stare Miasto S.A. in Konin

3. Centrum Logistyczno-Inwestycyjne Poznań Sp, z o.o. (CLIP) in Swarzędz bei Poznan

4. Euroterminal Slawkow

eine Spitzenleistung und das Ergebnis der Willenskraft der Gründer. Abbildung 3 zeigt die GVZ-Standorte.

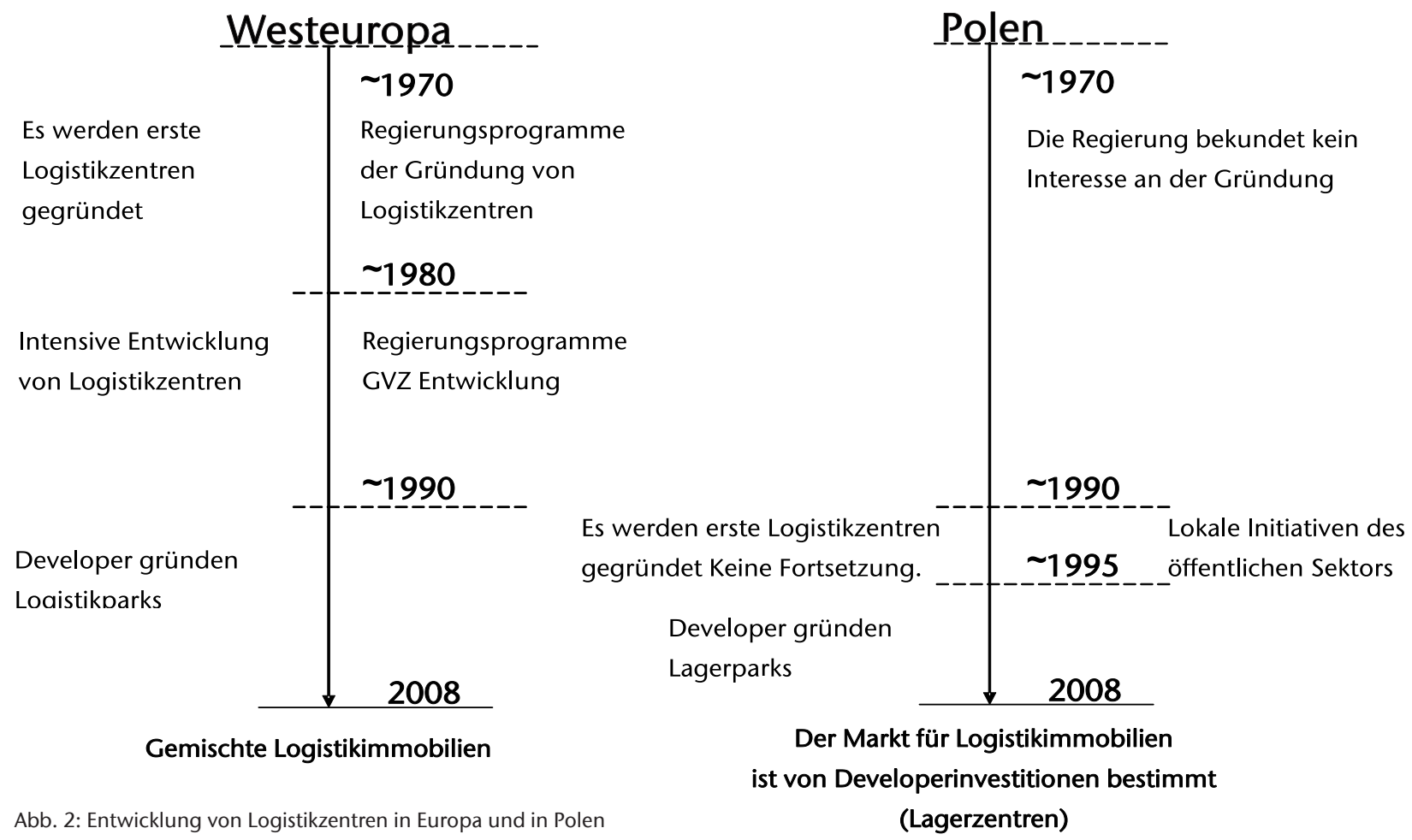




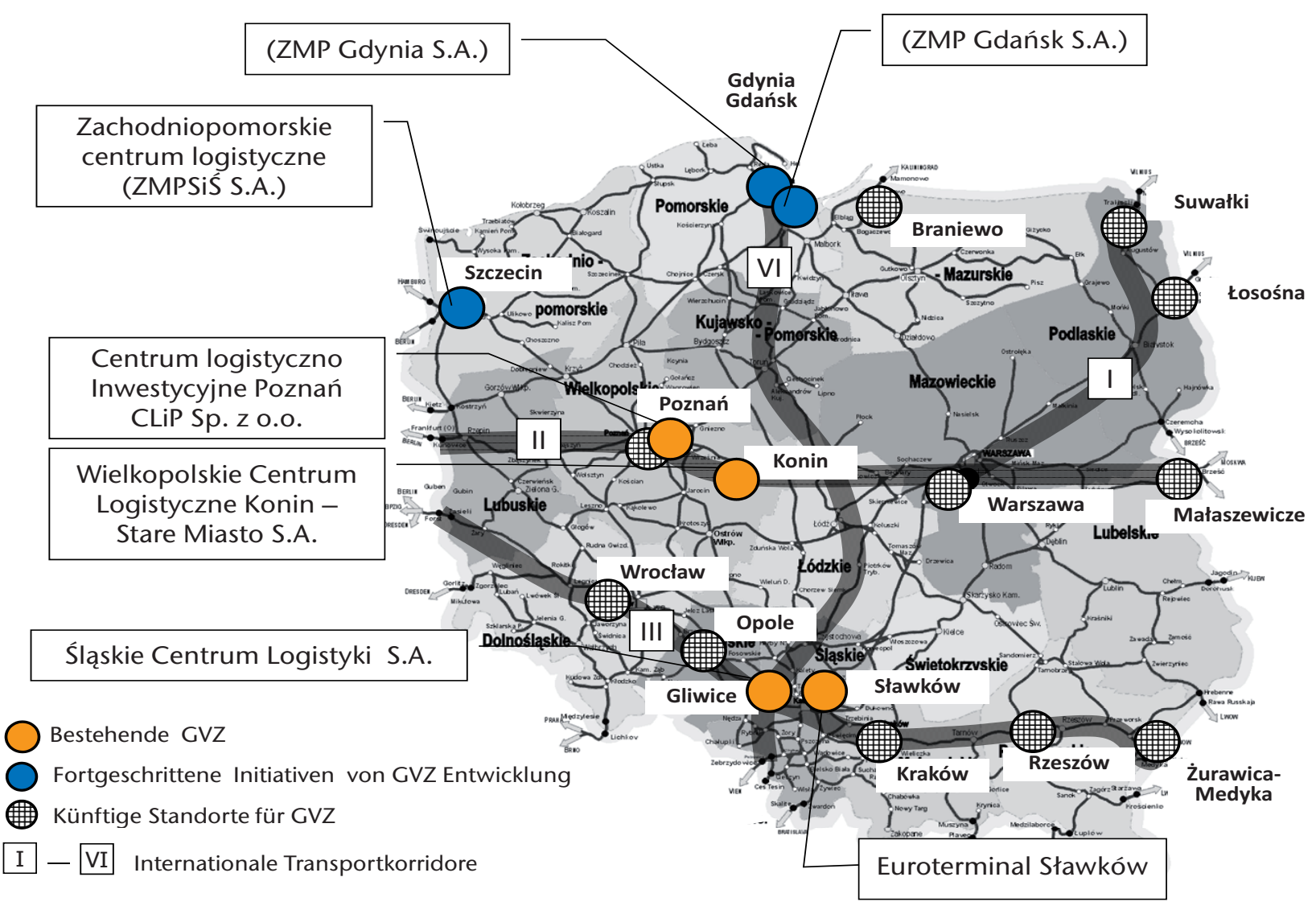

Abb. 3: GVZ Standorte in Polen, Quelle: Eigenstudie, Karte http:// www.pr.pkp.pl/

Besonders bemerkenswert ist die Entwicklung des Śląskie Centrum Logistyki SA in Gliwice. Dieses Logistikzentrum liegt im Bereich der Freihandelzone an der Kreuzung zweier Europäischen Transportkorridore: Berlin/Dresden-Wrocław-Lvov-Kiev (III) und GdańskKatowice-Zylin (IV). Der auf dem Gelände befindliche Binnenhafen wurde 1939 in Betrieb genommen. Auf der Liste der Investitionspläne stehen u. a. der Bau von $80.500 \mathrm{~m}^{2}$ Lagerfläche (geschlossen), $25500 \mathrm{~m}^{2}$ Lagerplatz (befestigt), Vergrößerung des Containerterminals bis zu 7,4 ha (Technische Daten des Terminals: Stellplatzkapazität $3.000 \mathrm{TEU}$, Jahresumschlag $60.000 \mathrm{TEU}$, 6 Bahngleise, 4 Reachstacker).

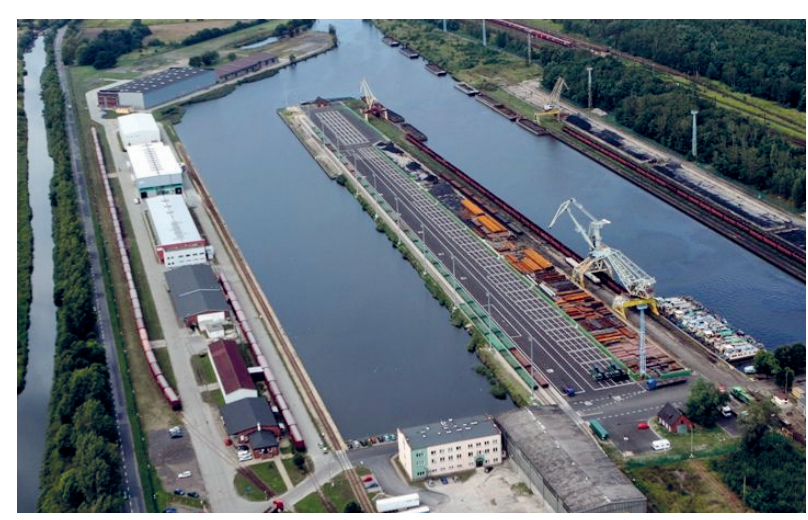

Abb. 4: GVZ in Gliwice
Andere moderne Logistikimmobilien entstehen vor allem in den fünf Woiwodschaften [1]: Mazowieckie, Łódzkie, Wielkopolskie, Śląskie und Dolnośląskie (Abb. 5). In diesen Woiwodschaften konzentrieren sich über 97\% der nach 1995 neu geschaffenen Lagerfläche.

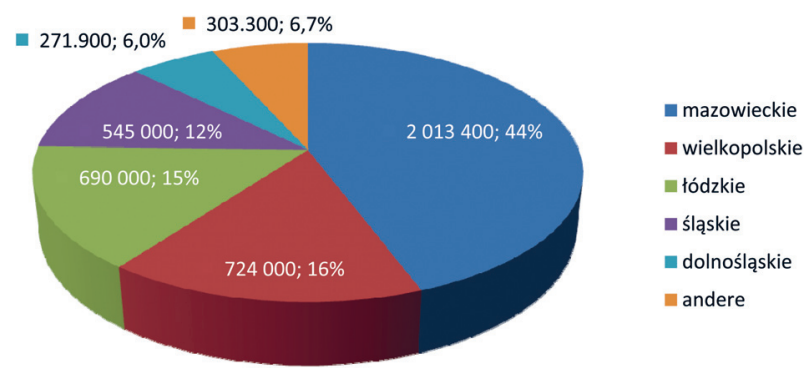

Abb 5: Moderne Lagerflächen in Polen 2008 (Logistyka Polsce 2007)

Über den Standort neuer Logistikimmobilien entscheiden vor allem folgende Faktoren: Größe der Ballungszentren, Einwohnerzahl, Kaufkraft, Charakter und Umfang der Produktion, Zugang zur Verkehrsinfrastruktur, wobei die Straßenanbindung den Vorzug hat. Die meisten Lagerobjekte in Polen haben keinen Anschluss an die Schienen-Infrastruktur.

Polen hat zahlreiche Vorteile, eine europäische Logistikregion mit einer großen Anzahl moderner Lager- 
objekte zu werden, jedoch gibt es auch Probleme und Widersprüche:

Paradoxon 1 - der schlechte Zustand der Straßeninfrastruktur begünstigt die Nachfrage an neuen Lagerflächen. Dieser Umstand steht nicht im Widerspruch zu der stattgefundenen Verbesserung der Verkehrsinfrastruktur, denn dieser Prozess verläuft nur allmählich und lokal. Wachsende Anforderungen der Unternehmen an Zuverlässigkeit und Servicegrad werden mit Problemen konfrontiert, die bei der Planung und Realisierung der Infrastruktur auftreten, denn durch den schlechten Zustand mehrerer Straßen in Polen werden termingerechte Lieferungen gestört. Aus diesem Grund müssen Bestände in Kundennähe gelagert werden, was einen Zuwachs an Lagerobjekten zur Folge hat.

Paradoxon 2 - durch eine geringe Integration der Lieferketten ist die Möglichkeit des Abbaus der Bestände nur beschränkt, was die wachsende Nachfrage an neuen Lagerflächen ebenfalls begünstigt.

\section{Ausblick}

Bis einschließlich 2008 haben Investoren - vor allem Developer wie: Prologis, Panattoni und Segro - über 4,5 Mio. Quadratmeter moderner Lagefläche in Polen bereitgestellt. Diese Zahl berücksichtigt jedoch nicht die Läger von Produktionsunternehmen, die für betriebseigene Zwecke errichtet werden. Schätzungen zufolge ist der Inlandsmarkt für Lagerflächen bei Weitem noch nicht gesättigt. Abhängig von der Entwicklung der polnischen Wirtschaft (BNP, Arbeitskosten, Wert der Landeswährung etc.) wird der Bedarf an neuen Lagerflächen in den kommenden fünf Jahren weitere 5 bis 7 Mio. $\mathrm{m}^{2}$ betragen. Diese werden jedoch aller Voraussicht nach nicht in den GVZ entstehen, sondern vor allem in monomodalen Lagerparks.

\section{Anmerkungen}

[1] Woiwodschaft ist ein polnischer Verwaltungsbezirk als oberste Stufe der territorialen Gliederung.

\section{Referenzen}

»Logistyka Polsce. Raport 2007 «. Instytut Logistyki i Magazynowania Poznań 2008.

\section{Literatur}

Fechner, I.; Przybycin, W. (2008): Rynek nieruchomości komercyjnych na cele budowy obiektów magazynowych i jego ograniczenia, LOGISTYKA 1/2008 Styczeń - Luty str. 14-16.

Fechner, I. (2007): Wpływ rozproszenia suprastruktury logistycznej na kształt krajowej sieci logistycznej, LOGISTYKA 3/2007 str. 20-22.

Fechner, I. (2007): Budowa nowych centrów logistycznych w Europie. XVI Międzynarodowa Konferencja Nowe trendy w transporcie i logistyce. Symbioza jakości transportu i ekologii. Uniwersytet Gdański Wydział Ekonomiczny Katedra Transportu i Logistyki. 12-13 czerwca 2007 r. Sopot.

Fechner, I.; Szyszka, G. (2007): Koncepcja rozwoju intermodalnego systemu infrastruktury transportowej. VII Międzynarodowa Konferencja TRANSLOG 2007 13-14 września 2007 Szczecin, Wydział Zarządzania i Ekonomiki Usług Uniwersytetu Szczecińskiego.

Fechner, I. (2007): Wpływ sektora publicznego na powodzenie budowy centrum logistycznego. II Konferencja Naukowo-Techniczna pt. Centra logistyczne w aspekcie zrównoważonego rozwoju regionów. Międzynarodowa Wyższa Szkoła Logistyki i Transportu we Wrocławiu. 26.10.2007 Wrocław.

Logistyka Polsce (2007): Logistyka Polsce. Raport 2007. Instytut Logistyki i Magazynowania Poznań.

\section{Autoren}

Dr.-Ing. Ireneusz Fechner

The Poznan School of Logistics

Institute of Logistics and Warehousing

Estkowskiego 6, 61-755 Poznan/Poland

Tel. +48 618504954, Ireneusz.fechner@wsl.com.pl

\section{Mag. Tomasz Janiak}

Institute of Logistics and Warehousing

Estkowskiego 6, 61-755 Poznań/Poland

Tel. +48 618504922, Tomasz.janiak@ilim.poznan.pl 